\title{
Usability Guidelines for Desktop Search Engines
}

\author{
Manuel Burghardt, Tim Schneidermeier, and Christian Wolff \\ Media Informatics Group, University of Regensburg, Regensburg, Germany \\ \{manuel.burghardt, tim.schneidermeier, christian.wolff\}@ur.de
}

\begin{abstract}
In this article we describe a usability evaluation of eight desktop search engines (DSEs). We used the heuristic walkthrough method to gather usability problems as well as individual strengths and weaknesses of the tested search engines. The results of the evaluation are integrated into a set of $30 \mathrm{de}-$ sign guidelines for user-friendly DSEs.
\end{abstract}

Keywords: usability testing, heuristic evaluation, desktop search engines, usability guidelines

\section{Introduction: Desktop Search Engines and the Case for Usability}

While web search engines (WSEs) have long since become a useful tool to fulfill everyday information needs on the web, so called desktop search engines (DSEs) are often inadequate to meet information needs on a user's private computer (Cole 2005). Strangely enough, many available systems do remarkably well in terms of performance and quality of search (Chang-Tien et al. 2007): That is why we believe that usability plays an important and oftentimes neglected role in the design of DSEs. Although there are many studies on the usability of WSE (Thurow \& Musica 2009) and guidelines for the general design for search user interfaces (Hearst 2009, Wilson 2012, Russell-Rose \& Tate 2013), the area of DSE has not been the subject of extensive usability research so far. While there are many similarities between web and desktop search, there are also some substantial differences: On the web, we usually search for new, mostly unseen information, but on our private desktops we search for "stuff we've seen before" (Dumais et al. 2003). The different information behavior for WSEs and DSEs (Bergman 2008) has implications for the design of a desktop search system, i.e. existing guidelines for the design of user-friendly web search systems (cf. Leavitt et al. 2006) cannot be adopted to the area of DSEs without further ado. In this paper we propose a set of guidelines for the design of user-friendly DSEs which has been derived from an expert-based usability inspection of existing desktop search systems.

adfa, p. 1, 2013.

(C) Springer-Verlag Berlin Heidelberg 2013 


\section{Evaluation Design}

The guidelines presented in this article are the result of a heuristic walkthrough (Sears 1997) for a total of eight available DSEs, including platform-specific systems such as Windows Search 4.0 and Apple's Spotlight, but also individual search engines such as Copernic or $x$ Friend (cf. Table 1 for an overview of all the DSEs that were subject of this evaluation study, cf. Figure 1 for an overview of the methodological approach). The heuristic walkthrough is an analytic evaluation method that relies on usability experts who perform an inspection of the subject of evaluation and document positive as well as negative usability and interaction issues. It is a combination of the rather unstructured, free-form heuristic evaluation (Nielsen 1994), and the more structured, task-based cognitive walkthrough (Wharton et al. 1994).

Table 1. Overview of all tested desktop search engines.

\begin{tabular}{|l|l|}
\hline Name & Source \\
\hline Apple Spotlight & part of the Apple operation system since Mac OS X 10.4 \\
\hline Archivarius 3000 & http://www.likasoft.com/de/document-search/index.shtml \\
\hline $\begin{array}{l}\text { Copernic Desktop } \\
\text { Search }\end{array}$ & http://www.copernic.com/en/products/desktop-search/index.html \\
\hline $\begin{array}{l}\text { Everything Search } \\
\text { Engine }\end{array}$ & http://www.voidtools.com/ \\
\hline Filehand Search & http://www.filehand.com/ \\
\hline Windows Search 4.0 & $\begin{array}{l}\text { http://www.microsoft.com/de-de/download/details.aspx?id=23 } \\
\text { (part of the MS Windows operation system since Windows 7) }\end{array}$ \\
\hline X1 & http://www.x1.com/ \\
\hline xFriend & http://www.xfriend.de/ \\
\hline
\end{tabular}

Each DSE was tested by an independent pair of evaluators ${ }^{1}$, each trying to accomplish a common information retrieval task with the respective search system. The evaluations revealed individual strengths, but also weaknesses of the respective DSE, which were documented as usability problems and categorized according to Nielsen's (1994) renowned framework, which consists of ten generic usability heuristics ${ }^{2}$ (cf. Table 2). In some cases, one identified usability problem was classified as violating several

${ }^{1}$ The evaluators were undergraduate students from the information science (http://iw.ur.de) and media informatics (http://mi.ur.de) B. A. degree programs at the University of Regensburg. They were trained in the heuristic walkthrough method in advance, and they also had knowledge about existing guidelines for the design of user-friendly WSEs (e.g. Leavitt et al. 2006 and Quirmbach 2012)

${ }^{2}$ Online version available at $<\mathrm{http} / /$ www.useit.com/papers/heuristic/heuristic_list.html $>$, last accessed on 1.10.2013. 
heuristics, which makes for a total of 70 usability problems ${ }^{3}$, and 92 violations of the usability heuristics in all.

Table 2. Overview of the heuristics used during the evaluation, and the number of violations for each heuristic.

\begin{tabular}{|ll|c|}
\hline Usability heuristic (Nielsen 1994) & Violations \\
\hline$(1) \quad$ Visibility of system status & 12 \\
\hline$(2) \quad$ Match between system and the real world & 9 \\
\hline$(3) \quad$ User control and freedom & 8 \\
\hline$(4) \quad$ Consistency and standards & 12 \\
\hline$(5) \quad$ Error prevention & 11 \\
\hline$(6) \quad$ Recognition rather than recall & 6 \\
\hline$(7) \quad$ Flexibility and efficiency of use & 7 \\
\hline$(8)$ & Aesthetic and minimalist design & 8 \\
\hline$(9)$ & Help users recognize, diagnose, and recover from errors & 9 \\
\hline$(10)$ & Help and documentation & 10 \\
\hline \multicolumn{2}{l}{} & Total: 92 \\
\cline { 2 - 2 }
\end{tabular}

After the heuristic walkthrough, we clustered some of the redundant usability problems and also considered specific strengths which had been evaluated positively by the testers. We integrated the reported problems as well as the positive aspects of the evaluated DSEs into an initial set of 36 usability guidelines.

In a next step, these guidelines were discussed with the participants of the previous heuristic walkthrough in order to get feedback on the appropriateness of the suggested guidelines for the respective DSE they had tested before. The group discussion revealed some interesting wording problems and also showed that some guidelines were formulated redundantly or put in the wrong heuristic category. After this first, theoretical validation round we reduced our set of guidelines to a total of 30 , and also did some rephrasing to make them more comprehensible. The final set of guidelines is presented in the next section.

\footnotetext{
${ }^{3}$ We do not report the exact number of usability problems for each DSE, as the evaluation was not about comparing several systems to find out which one is best, but rather to analyze why a system is bad with regards to its usability.
} 


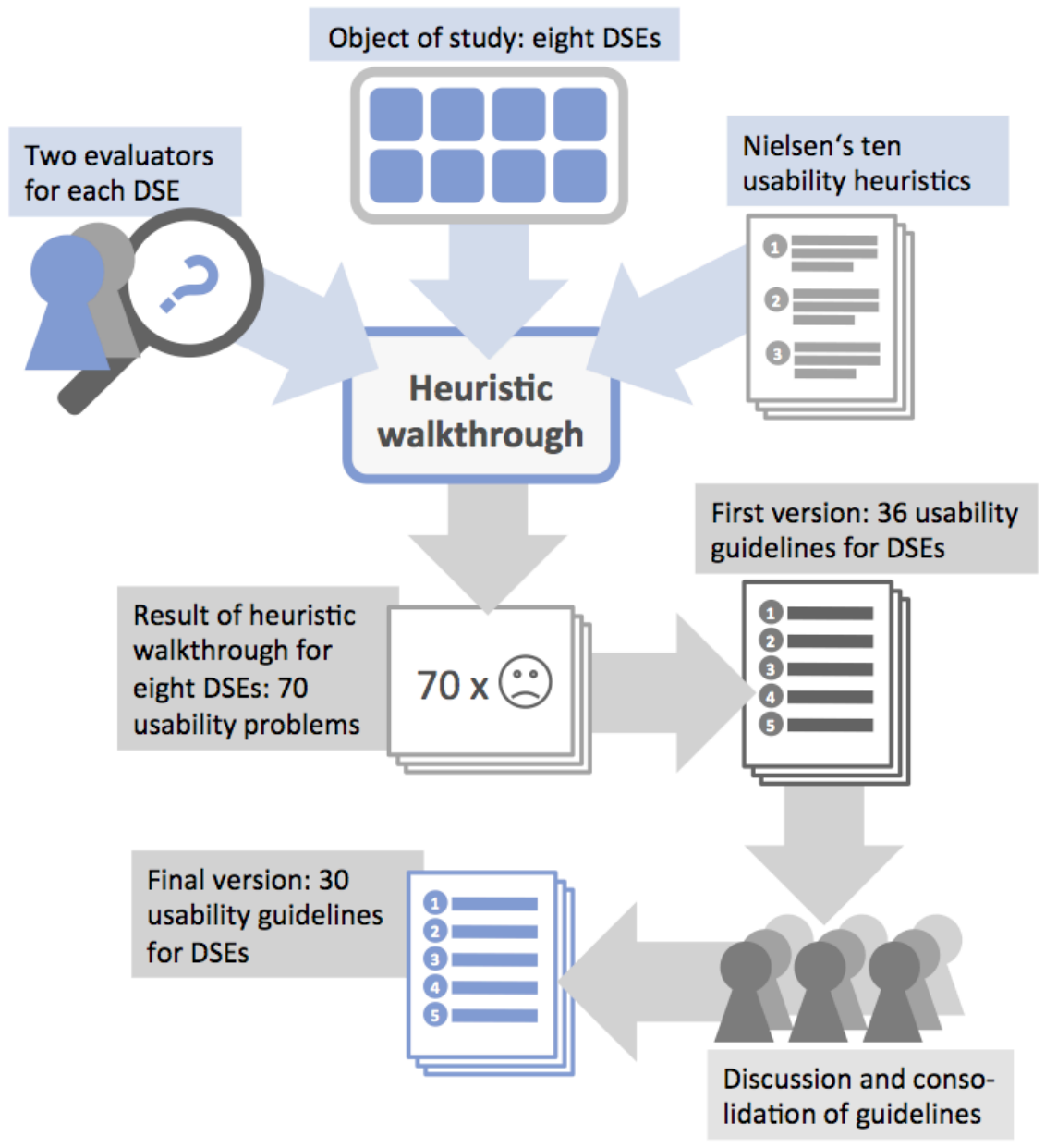

Fig. 1. Overview of the methodological approach used to gather usability guidelines for DSEs.

\section{Guidelines for the Design of User-Friendly Desktop Search Engines}

This section presents the results of a heuristic walkthrough for eight DSEs: a set of 30 guidelines for the design of user-friendly search engines for the desktop context, categorized according to Nielsen's (1994) ten usability heuristics.

Visibility of system status | The guidelines in this category ensure that the user of a DSE knows what the software is doing at a given point in time. These guidelines are all about comprehensibility and transparency of the system status, which includes appropriate feedback on specific operations and the coming about of certain results. 
- G1.1 It should be obvious to the user which areas of the system are indexed and thus included in the search scope at a given point in time.

- G1.2 It should be obvious to the user when the DSE is busy, building or updating the index, which may have effects on search performance.

- G1.3 It should be obvious to the user how many files are actually searched, and how many relevant documents were finally retrieved.

Match between system and the real world | The DSE should speak the language of the user, i.e. it should use words and metaphors the user is already familiar with. Information, especially on the results list, should be presented in an intuitive and comprehensible order.

- G2.1 If there is an explicit button to submit a query, it should be labeled unambiguously with "search".

- G2.2 If there are identical documents in the results list, i.e. documents that are stored redundantly on different locations of the system, these should be marked as duplicates.

- G2.3 On the results list, it should be obvious for the user where the respective documents are stored (make the document path available).

User control and freedom | The user should be free to control certain functionalities of the DSE. For the designer, this is often a tightrope walk between hiding certain functions to reduce complexity, but at the same time provide access to these functions at any point in time, to ensure full user control and freedom.

- G3.1 If necessary, the user should be able to access advanced search options, which are hidden per default to reduce unnecessary complexity in a simple and intuitive way.

- G3.2 The user should be able to define the scope of the search in a simple and intuitive way.

- G3.3 If a search takes exceptionally long, the user should be able to cancel it manually.

- G3.4 If necessary, the user should be able to filter the list of results by different criteria such as file format, date or name.

Consistency and standards $\mid$ The DSE should be consistent in itself with regard to design and wording as well as functionality and operations. It should also adhere to standards that are known from typical desktop applications and to conventions that have been established in the area of WSE.

- G4.1 Standards and conventions that are known from the area of desktop applications (e.g. design of icons or menus) should be observed.

- G4.2 Colors, layout and font should be used in a consistent way.

- G4.3 Basic user interface elements, like for instance the search field or the search button, should be used consistently in different situations.

- G4.4 The DSE should support truncation and search operators (e.g. AND / OR / NOT), or their conventionalized shortcuts, that may be known from WSEs. 
Error prevention | This category contains guidelines for a pre-emptive system design that reduces potential interaction errors.

- G5.1 The user should not be required to set any advanced search parameters to enter a simple query.

- G5.2 If the user has not configured it otherwise, the DSE should search all areas of the system on default.

- G5.3 The DSE should prevent the user from misspelling the query and actively support query formulation by providing search as you type functionality as known from many WSEs.

- G5.4 The DSE should translate special characters on the query side as well as on the indexing side into the platform specific encoding.

Recognition rather than recall | The DSE should support recognition rather than recall, which first and foremost is relevant for the query a user formulates at the beginning of his search. After submitting the query to the DSE, the user usually sees a results page: To evaluate the results, it is vital to compare it with the original query, thus the query should be visible at this stage of search.

- G6.1 The DSE should display the original query on the results page.

- G6.2 The search field should be big enough to display complex queries.

Flexibility and efficiency of use | The search engine should provide shortcuts that accelerate the interaction for experts, but do not obfuscate novice users. Frequently used functions should be easily accessible for any user group.

- G7.1 The user should be able to open the search dialogue at any time with a single action (e.g. a mouse click or a keyboard shortcut).

- G7.2 The user should be able to save complex queries for later reuse (personalized search history).

- G7.3 The user should be able to jump to the target documents directly by clicking on the items in the results list.

- G7.4 A query should be processed in less than two seconds, as the user is accustomed to high speed information retrieval from the web context.

Aesthetic and minimalist design | Interaction should be kept simple, i.e. the interface should not contain irrelevant or information used rarely.

- G8.1 The items in the results list should hide inadequate metadata from the user.

- G8.2 The user interface is functional and only consists of the necessary interaction components, such as search field, search button, and advanced search options.

Help users recognize, diagnose, and recover from errors | If an error occurs, the DSE should provide feedback on the problem cause, and also suggest a potential solution. In the case of DSE, the most frequent problems that occur are queries which do not return any results. 
- G9.1 If a query returns zero results, the DSE should provide hints on how to reformulate or improve the unsuccessful query.

Help and documentation | Ideally, a user should be able to use the basic functions of a DSE without having to read a manual or a documentation. The DSE should be selfexplanatory or provide useful information to understand complex interaction behavior (e.g. in the form of tooltips or meaningful icons). Nevertheless, advanced and complex functions should be described in some kind of documentation. The documentation should be easy to use, and focus on potential user problems and questions.

- G10.1 The DSE should describe and explain the advanced search options.

- G10.2 There should be a document that describes and explains the indexing function in detail.

- G10.3 The DSE should provide search templates (i.e. exemplary queries) that illustrate how to use the search syntax to formulate successful queries.

\section{Outlook}

We believe that our set of guidelines can help future developers of DSEs to build more usable systems, and that it may serve as an evaluation tool for users who have to decide which system to choose from the abundance of available search engines. It must be stressed, however, that the guidelines presented here are not meant to be exhaustive for any usability aspect of DSEs, but rather serve as a basic collection of hints and guidelines, that may be adjusted and extended for specific scenarios if necessary. In addition, the concept of desktop search engines needs some serious reconsideration, as the traditional desktop metaphor is slowly fading away in the face of ubiquitous and mobile computing, and the increasing availability of cloud storage services. We are planning to further validate, extend and refine the usability guidelines for DSEs, and to eventually adapt them for the domain of mobile search (Tan et al. 2008) in consecutive studies.

\section{Bibliography}

Bergman, O., Beyth-Marom, R., Nachmias, R., Gradovitch, N., Whittaker, S.: Improved search engines and navigation preference in personal information management. In: ACM Transactions on Information Systems (TOIS), Vol. 26/4 (2008), pp. 1-24.

Chang-Tien, L., Shukla, M., Subramanya, S. H., Yamin, W.: Performance Evaluation of Desktop Search Engines. IEEE International Conference on Information Reuse and Integration (2007), pp. 110-115.

Cole, B.: Search engines tackle the desktop. IEEE Computer, Vol. 38/3 (2005), pp.14-17.

Dumais, S., Cutrell, E., Cadiz, J. J., Jancke, G., Sarin, R., Robbins, D. C.: Stuff I've seen: a system for personal information retrieval and re-use. Proceedings of the 26th annual international ACM SIGIR conference on Research and development in information retrieval (2003), pp. 72-79. 
Hearst, M. A.: Search User Interfaces. Cambridge University Press (2009).

Leavitt, M. et al.: Research-Based Web Design and Usability Guidelines. U.S. Dept. of Health and Human Services (2006).

Nielsen, J.: Heuristic evaluation. In: Nielsen J., Mack, R. (eds.): Usability Inspection Methods. John Wiley \& Sons, New York (1994), pp. 25-62.

Russell-Rose, T., Tate, T.: Designing the Search Experience. The Information Architeture of Discovery. Morgan Kaufman, Waltham, MA (2013).

Sears, A.: Heuristic walkthroughs: finding the problems without the noise. In: International Journal of Human-Computer Interaction, 9(3) (1997), pp. 213-234.

Tan, C., Sheng, B., Wang, H., Li, Q.: Microsearch: When search engines meet small devices. Pervasive Computing, Lecture Notes in Computer Science, Vol. 5013 (2008), pp. 93-110.

Thurow, S. \& Musica N.: When Search Meets Web Usability. New Riders, Indianapolis, IN (2009).

Quirmbach, S.: Suchmaschinen, X-media-press. Springer, Heidelberg (2012).

Wharton, C., Rieman, J., Lewis, C., Polson, P.: The cognitive walkthrough method: A practitioner's guide. In: Nielsen J., Mack, R. (eds.): Usability Inspection Methods. John Wiley \& Sons (1994), pp. 105-140.

Wilson, M. L.: Search User Interface Design. Morgan \& Claypool Publishers, San Francisco (2012). 